\title{
LMA effectively single-mode thulium doped fibre with normal dispersion at wavelengths around $2 \mathrm{um}$
}

\author{
C. Baskiotis*, A.M. Heidt, S. Alam and D.J. Richardson \\ Optoelectronics Research Centre, University of Southampton, Southampton, SO17 1BJ, United Kingdom \\ *cb5y10@orc.soton.ac.uk
}

During the last few years, an increasing interest in Large Mode Area fibre operating around a wavelength of $2 \mu \mathrm{m}$ for high-power fibre laser applications has been observed. For most pulsed laser applications, it is desirable to operate the fibre in the normal dispersion regime to avoid the limiting effects of modulational instability (MI)/soliton formation [1]. However, it is very difficult to achieve normal dispersion in conventional step-index large mode area fibres operating around a wavelength of $2 \mu \mathrm{m}$ due to the fact that the dispersion of their fundamental mode is mainly dominated by the material dispersion of silica, which is anomalous and very high around this wavelength (e.g.: 44ps/(nm.km) at 1930nm, cf. Fig. 1.(b)).

To obtain a normally dispersive LMA gain fibre for $2 \mu \mathrm{m}$, we propose a structure consisting of a thulium doped step-index core of diameter $\mathrm{d}_{\mathrm{c}}=29.6 \mu \mathrm{m}$ and of refractive index difference as compared to silica $(\Delta \mathrm{n})$ of $3 \times 10^{-3}$, surrounded by one ring of highly-doped Germanium rods $\left(\Delta \mathrm{n} \sim 30 \times 10^{-3}\right)$ with a nearly parabolic refractive index profile (cf. Fig. 1.(a)). The arrangement of the Germanium rods is identical to those obtained in the case of a 7-cell core photonic bandgap fibre with a triangular lattice in its cladding (cf. Fig. 1.(a)). We use the Finite Element Method in combination with the Marcuse model [2] to perform simulations of this structure, when it is bent at a representative bend radius of $10 \mathrm{~cm}$.

Normal dispersion over the wavelength range $[1895 \mathrm{~nm} ; 1950 \mathrm{~nm}]$ is obtained for the fundamental core mode, thanks to resonant couplings with the modes of the highly-doped Germanium rods (cf. Fig. 1.(b)). Moreover, negligible bend losses (below $1 \mathrm{~dB} / \mathrm{km}$ ) and an Effective Area larger than $600 \mu \mathrm{m}^{2}$ are achieved over the whole wavelength range (cf. Fig. 1.(c)).

All the higher-order core modes exhibit bend losses larger than $50 \mathrm{~dB} / \mathrm{m}$ over the full normal dispersion range, except the first higher order core mode $\mathrm{LP}_{11}$. However, in the wavelength range $[1915 \mathrm{~nm} ; 1945 \mathrm{~nm}]$, the percentage of power of the $\mathrm{LP}_{11}$ core mode in the core is lower than $35 \%$, while the percentage of power of the fundamental mode in the core is larger than $80 \%$ (cf. Fig. 1.(d-f)). It is therefore reasonable to assume that the $\mathrm{LP}_{11}$ core mode will experience significantly lower gain relative to the fundamental core mode under normal operating conditions allowing effective single mode operation to be achievable.

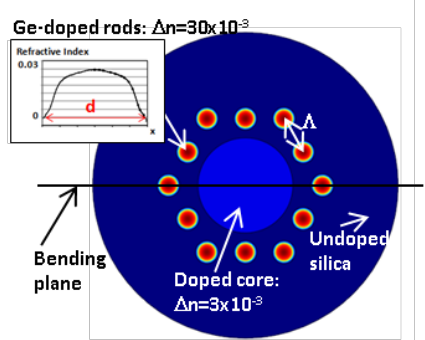

(a)

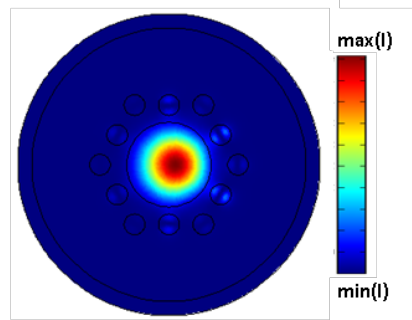

(d)

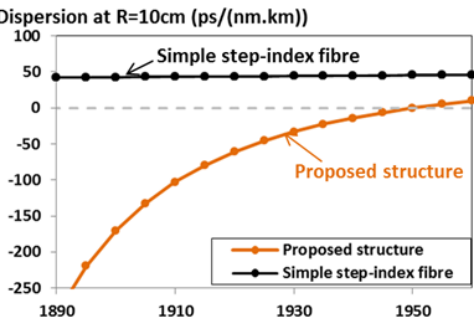

(b)

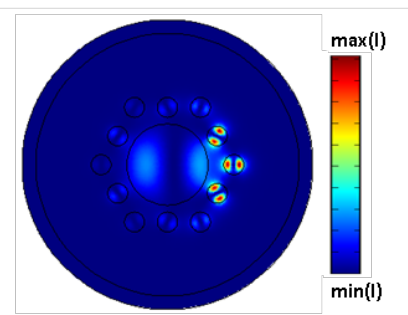

(e)

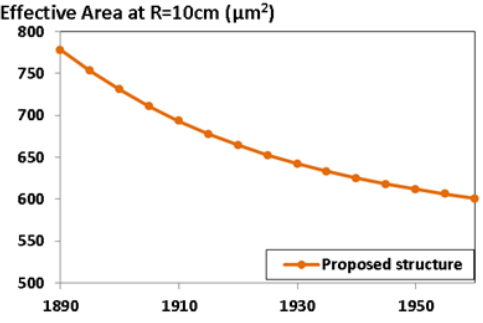

(c)

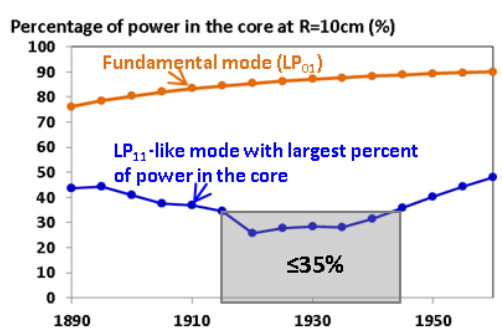

(f)

Fig. 1 (a) Schematic of the proposed fibre. The thulium doped core has a representative $\Delta \mathrm{n}$ of $3 \times 10^{-3}$, the Germanium rods have a nearly parabolic profile of maximum $\Delta \mathrm{n}$ of $\sim 30 \times 10^{-3}$ (cf. inset), the rod diameter d is of

$7.2 \mu \mathrm{m}$ and the pitch is $\Lambda=12 \mu \mathrm{m}$. The doped core diameter is $29.6 \mu \mathrm{m}$. (b-c) Fundamental core-mode (LP $\mathrm{L}_{01}$ ) dispersion (b) and Effective area (c) as a function of the wavelength, when the fibre is bent at a radius $\mathrm{R}$ of $10 \mathrm{~cm}$. (d-e) Intensity profile of the $\mathrm{LP}_{01}$ core mode (d) and the $\mathrm{LP}_{11}$ core mode presenting the largest amount of power in the core (e), when the fibre is bent at $R=10 \mathrm{~cm}$ at the wavelength of $1945 \mathrm{~nm}$. (f) Percentage of power in the core of the LP $\mathrm{P}_{01}$ core mode and the $\mathrm{LP}_{11}$ core mode presenting the largest amount of power in the core when the fibre is bent at $\mathrm{R}=10 \mathrm{~cm}$.

\section{References}

[1] A. Chong, J. Buckley, W. Renninger, and F. Wise, "All-normal-dispersion femtosecond fiber laser," Opt. Express 14, 10095-10100 (2006).

[2] D. Marcuse, "Influence of curvature on the losses of doubly clad fibers," Appl. Opt. 21, 4208-4213 (1982). 